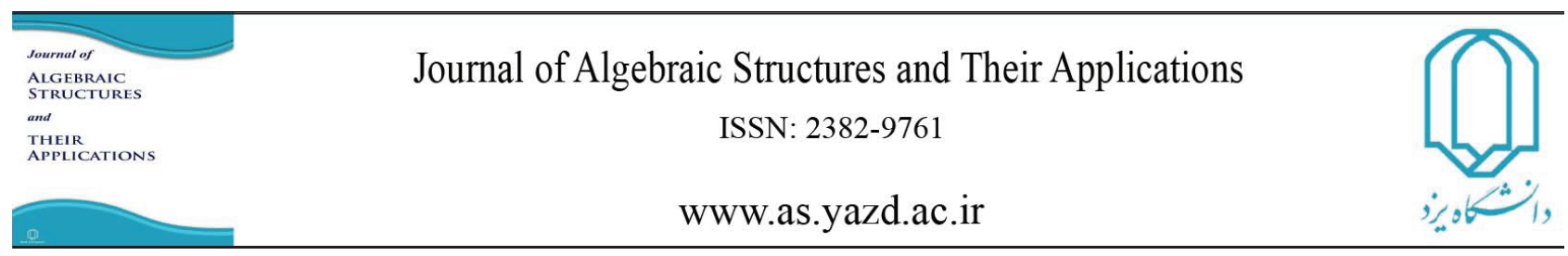

Algebraic Structures and Their Applications Vol. 5 No. 1 ( 2018 ) pp 41-49.

\title{
A SHORT NOTE ON PRIME SUBMODULES
}

\author{
JAFAR A'ZAMI
}

Communicated by B. Davvaz

\begin{abstract}
Let $R$ be a commutative ring with identity and $M$ be a unital $R$-module. A proper submodule $N$ of $M$ with $N:_{R} M=\mathfrak{p}$ is said to be prime or $\mathfrak{p}$-prime (p a prime ideal of $R$ ) if $r x \in N$ for $r \in R$ and $x \in M$ implies that either $x \in N$ or $r \in \mathfrak{p}$. In this paper we study a new equivalent conditions for a minimal prime submodules of an $R$-module to be a finite set, whenever $R$ is a Noetherian ring. Also we introduce the concept of arithmetic rank of a submodule of a Noetherian module and we give an upper bound for it.
\end{abstract}

\section{INTRODUCTION}

Throughout this paper, let $R$ be a commutative ring (with identity) and $M$ be a unital $R$-module. A proper submodule $N$ of $M$ with $N:_{R} M=\mathfrak{p}$ is said to be prime or $\mathfrak{p}$-prime (p a prime ideal of $R$ ) if $r x \in N$ for $r \in R$ and $x \in M$ implies that either $x \in N$ or $r \in \mathfrak{p}$. An other equivalent notion of prime submodules was first introduced and systematically studied in [6]. Prime submodules have been studied by several authors; see, for example, [4], [T], [7], [9], [I0], [II], [I2] and [14]. In section 2, we prove some new results about the finiteness of the set of DOI : http://dx.doi.org/10.29252/asta.5.1.41

MSC(2010): Primary: 13C05; Secondary: 13E15

Keywords: arithmetic rank of a submodule, associated primes, height of a prime submodule, minimal prime submodule, prime submodule.

Received: 09 November 2017, Accepted: 01 September 2018.

(C) 2018 Yazd University. 
minimal prime submodules of an $R$-module. Also we introduce the concept of arithmetic rank of a submodule of a Noetherian module and we give an upper bound for it. Throughout, for any ideal $\mathfrak{b}$ of $R$, the radical of $\mathfrak{b}$, denoted by $\operatorname{Rad}(\mathfrak{b})$, is defined to be the set $\left\{x \in R: x^{n} \in \mathfrak{b}\right.$ for some $n \in \mathbb{N}\}$ and we denote $\{\mathfrak{p} \in \operatorname{Spec}(R): \mathfrak{p} \supseteq \mathfrak{b}\}$ by $V(\mathfrak{b})$, where $\operatorname{Spec}(R)$ denotes the set of all prime ideals of $R$. The symbol $\subseteq$ denotes containment and $\subset$ denotes proper containment for sets. If $N$ is a submodule of $M$, we write $N \leq M$. We denote the annihilator of a factor module $M / N$ of $M$ by $\left(N:_{R} M\right)$. The set of all maximal ideals of $R$ is denoted by $\operatorname{Max}(R)$. For any unexplained notation and terminology we refer the reader to [5], [1:3] and [16].

\section{Preliminaries}

The results of this section which will be useful in the next section given in [2].

Proposition 2.1. Let $R$ be ring and $M$ be a non-zero $R$-module and $N$ be a submodule of $M$. Let $\mathfrak{p}_{1}, \ldots, \mathfrak{p}_{n}$ be distinct prime ideals of $R$. Let for each $1 \leq i \leq n, N_{i}$ be a $\mathfrak{p}_{i}$-prime submodule of $M$. If $N \subseteq \cup_{i=1}^{n} N_{i}$, then $N \subseteq N_{j}$ for some $1 \leq j \leq n$.

Proof. We do induction on $n$. The case $n=2$ is easy. Now let $n \geq 3$ and the case $n-1$ is settled. By definition for each $1 \leq i \leq n$ we have $\mathfrak{p}_{i}=\left(N_{i}:_{R} M\right)$. From the hypothesis $N \subseteq \cup_{i=1}^{n} N_{i}$ it follows that $N=\cup_{i=1}^{n}\left(N_{i} \cap N\right)$. Now let the contrary be true. Then $N \nsubseteq N_{i}$ and hence $\left(N_{i} \cap N\right) \neq N$, for any $1 \leq i \leq n$. Also, from the inductive hypothesis it follows that $N \neq \cup_{i \in(\{1, \ldots, n\} \backslash\{k\})}\left(N_{i} \cap N\right)$ for each $1 \leq k \leq n$ and so $\left(N_{k} \cap N\right) \not \subset \cup_{i \in(\{1, \ldots, n\} \backslash\{k\})}\left(N_{i} \cap N\right)$. Let $\mathfrak{q}$ be a minimal element of the set $\left\{\mathfrak{p}_{1}, \ldots, \mathfrak{p}_{n}\right\}$ with respect to " $\subseteq$ ". Then $\mathfrak{p}_{i} \nsubseteq \mathfrak{q}$ for each $\mathfrak{p}_{i} \in\left(\left\{\mathfrak{p}_{1}, \ldots, \mathfrak{p}_{n}\right\} \backslash\{q\}\right)$. Without loss of generality we may assume that $\mathfrak{q}=\mathfrak{p}_{n}$. Let $J_{i}:=\left(N_{i}:_{R} N\right)$, for all $i=1, \ldots, n$. Then from the definition it follows that $\mathfrak{p}_{i} \subseteq J_{i}$, for all $i=1, \ldots, n$. On the other hand for each $x \in N$ and $r \in R$, if $r x \in\left(N_{i} \cap N\right)$ and $x \notin\left(N_{i} \cap N\right)$, then $r x \in N_{i}$ and $x \notin N_{i}$. Therefore it follows from the definition that $r \in \mathfrak{p}_{i}$. So $r M \subseteq N_{i}$, and consequently, $r N \subseteq\left(N_{i} \cap N\right)$. As $\left(N_{i} \cap N\right) \neq N$ it follows that there exists an element $y \in\left(N \backslash\left(N_{i} \cap N\right)\right)$. Now for each $s \in J_{i}$ we have $s y \in\left(N_{i} \cap N\right) \subseteq N_{i}$ and $y \notin N_{i}$. So it follows from the definition that $s \in \mathfrak{p}_{i}$. Therefore, $\left(N_{i}:_{R} N\right)=J_{i}=\mathfrak{p}_{i}=\left(N_{i}:_{R} M\right)$. But it is easy to see that $\left(N_{i}:_{R} N\right)=\left(\left(N_{i} \cap N\right):_{R} N\right)$. Thus for each $1 \leq i \leq n, N_{i} \cap N$ is a $\mathfrak{p}_{i}$-prime submodule of $N$. Therefore without loss of generality we may assume that $N=M=\cup_{i=1}^{n} N_{i}$ and $N_{n} \nsubseteq \cup_{i=1}^{n-1} N_{i}$. Next let $T:=\cap_{i=1}^{n} N_{i}$. Then it is not to see that for each $1 \leq i \leq n, N_{i} / T$ is a $\mathfrak{p}_{i}$-prime submodule of $M / T$ and $M / T=\cup_{i=1}^{n} N_{i} / T$. Therefore, without loss of generality we may assume $M=\cup_{i=1}^{n} N_{i}$ and $\cap_{i=1}^{n} N_{i}=0$ and $N_{n} \nsubseteq \cup_{i=1}^{n-1} N_{i}$. Then there is an exact sequence $0 \rightarrow M \rightarrow \oplus_{i=1}^{n} M / N_{i}$, which implies that $\cap_{i=1}^{n} \mathfrak{p}_{i}=\operatorname{Ann}_{R}\left(\oplus_{i=1}^{n} M / N_{i}\right) \subseteq \operatorname{Ann}_{R}(M)$. On the other hand for each $1 \leq i \leq n$ we have $\operatorname{Ann}_{R}(M) \subseteq\left(N_{i}: R M\right)=\mathfrak{p}_{i}$. So $\operatorname{Ann}_{R}(M) \subseteq \cap_{i=1}^{n} \mathfrak{p}_{i}$. Hence $\operatorname{Ann}_{R}(M)=\cap_{i=1}^{n} \mathfrak{p}_{i}$. Now if we 
have $\cap_{i=1}^{n-1} N_{i}=0$, then there is an exact sequence $0 \rightarrow M \rightarrow \oplus_{i=1}^{n-1} M / N_{i}$, which implies that $\cap_{i=1}^{n-1} \mathfrak{p}_{i}=\operatorname{Ann}_{R}\left(\oplus_{i=1}^{n-1} M / N_{i}\right) \subseteq \operatorname{Ann}_{R}(M)=\cap_{i=1}^{n} \mathfrak{p}_{i} \subseteq \mathfrak{p}_{n}$. So $\mathfrak{p}_{t} \subseteq \mathfrak{p}_{n}$, for some $1 \leq t \leq n-1$, which is a contradiction. So $\cap_{i=1}^{n-1} N_{i} \neq 0$. Then there is an element $0 \neq a \in \cap_{i=1}^{n-1} N_{i}$. As $\cap_{i=1}^{n} N_{i}=0$, it follows that $a \notin N_{n}$. On the other hand since $N_{n} \nsubseteq \cup_{i=1}^{n-1} N_{i}$, it follows that there is an element $b \in N_{n}$ such that $b \notin \cup_{i=1}^{n-1} N_{i}$. Now as $a+b \in \cup_{i=1}^{n} N_{i}$, it follows that $a+b \in N_{k}$ for some $1 \leq k \leq n$, which is a contradiction. This completes the inductive step. $\square$

Remark: Proposition 2.1 does not hold in general. For example let $p \geq 2$ be a prime number and $2 \leq n \in \mathbb{N}$. Let $R=\mathbb{Z}_{p}=\{\overline{0}, \overline{1}, \ldots, \overline{p-1}\}$ and $M=\oplus_{i=1}^{n} \mathbb{Z}_{p}$. Let

$$
\mathfrak{A}=\{N: N=R x, \text { for some } 0 \neq x \in M .\} .
$$

Then $\mathfrak{A}$ is a finite set that has at most $2^{p^{n}}$ element and for each $N \in \mathfrak{A}, N$ is a $\{\overline{0}\}$-prime submodule of $M$ such that $M \subseteq \cup_{N \in \mathfrak{A}} N$. But $M \nsubseteq N$ for any $N \in \mathfrak{A}$.

The following proposition is a generalization of [1.3, Ex. 16.8].

Proposition 2.2. Let $R$ be a ring, $M$ a non-zero $R$-module, $N$ a submodule of $M$ and $x \in M$. Let $\mathfrak{p}_{1}, \ldots, \mathfrak{p}_{n}$ be distinct prime ideals of $R$. Let for each $1 \leq i \leq n, N_{i}$ be a $\mathfrak{p}_{i}$-prime submodule of $M$. If $N+R x \nsubseteq \cup_{i=1}^{n} N_{i}$, then there exists $a \in N$ such that $a+x \notin \cup_{i=1}^{n} N_{i}$.

Proof. We use induction on $n$. Let $n=1$. If $x \in N_{1}$ then $N \nsubseteq N_{1}$. So there is $a \in N \backslash N_{1}$ and it is easy to see that $a+x \notin N_{1}$. But if $x \notin N_{1}$, then by choosing $a=0 \in N$ the assertion holds. Now suppose $n \geq 2$ and the case $n-1$ is settled. Let $\mathfrak{q}$ be a minimal element of the set $\left\{\mathfrak{p}_{1}, \ldots, \mathfrak{p}_{n}\right\}$ with respect to " $\subseteq$ ". Then $\mathfrak{p}_{i} \not \subseteq \mathfrak{q}$ for each $\mathfrak{p}_{i} \in\left(\left\{\mathfrak{p}_{1}, \ldots, \mathfrak{p}_{n}\right\} \backslash\{q\}\right)$. Without loss of generality we may assume that $\mathfrak{q}=\mathfrak{p}_{n}$. Then it is easy to see that $\cap_{i=1}^{n-1} \mathfrak{p}_{i} \not \subset \mathfrak{p}_{n}$. By inductive hypothesis there is an element $b \in N$ such that $b+x \notin \cup_{i=1}^{n-1} N_{i}$. So the assertion hold for $a=b$, whenever $b+x \notin N_{n}$. So we may assume $b+x \in N_{n}$. Then we claim that $N \nsubseteq N_{n}$. Because, if $N \subseteq N_{n}$ then $x \in N_{n}$ and so $N+R x \subseteq N_{n} \subseteq \cup_{i=1}^{n} N_{i}$, which is a contradiction. Therefore, there exists an element $c \in N \backslash N_{n}$. As $\cap_{i=1}^{n-1} \mathfrak{p}_{i} \not \subset \mathfrak{p}_{n}$ it follows that there exists an element $r \in\left(\cap_{i=1}^{n-1} \mathfrak{p}_{i}\right) \backslash \mathfrak{p}_{n}$. Then it easily follows from the definition of the $\mathfrak{p}_{n}$-prime submodule that $r c \notin N_{n}$. Moreover, since $r \in \cap_{i=1}^{n-1} \mathfrak{p}_{i}$ it follows from the definition that $r c \in \cap_{i=1}^{n-1} N_{i}$. Now it is easy to see that $r c+b+x \notin \cup_{i=1}^{n} N_{i}$. Therefore, the assertion hold for $a:=r c+b \in N$. This completes the induction step. 
Definition 2.3. Let $R$ be a Noetherian ring and $M$ be a finitely generated $R$-module. For each $\mathfrak{p}$-prime submodule $N$ of $M$ we define $\mathfrak{p}$-height of $N$ as:

$$
\mathfrak{p}-\operatorname{ht}(N):=\sup \left\{k \in \mathbb{N}_{0}: \exists N_{0} \subset \cdots \subset N_{k}=N, \text { with } N_{i} \in \operatorname{Spec}_{R}^{\mathfrak{p}}(M), \forall i\right\},
$$

where $\operatorname{Spec}_{R}^{\mathfrak{p}}(M)$ denotes to the set of all $\mathfrak{p}$-prime submodules of $M$ as an $R$-module.

Definition 2.4. Let $R$ be a Noetherian ring and $M$ be a finitely generated $R$-module. For each p-prime submodule $N$ of $M$ we define height of $N$ as:

$$
\operatorname{ht}(N):=\sup \left\{k \in \mathbb{N}_{0}: \exists N_{0} \subset \cdots \subset N_{k}=N, \text { with } N_{i} \in \operatorname{Spec}_{R}(M), \forall i\right\},
$$

where $\operatorname{Spec}_{R}(M)$ denotes to the set of all prime submodules of $M$ as an $R$-module.

\section{Minimal PRIMe SUbModules}

The following lemma is needed in the proof of the first main result of this section. Note that in the sequel for any submodule $B$ of an $R$-module $M$, the set of all minimal prime submodules of $M$ over $B$ is denoted by $\operatorname{Min}(B)$. Moreover, we denote $\operatorname{Min}(0)$ by $\operatorname{Min}(M)$. Also, $V(B)$ is defined as follows:

$$
V(B):=\left\{N \in \operatorname{Spec}_{R}(M): N \supseteq B\right\}
$$

Lemma 3.1. Let $R$ be a commutative ring and $\mathfrak{p}, \mathfrak{q} \in \operatorname{Spec}(R)$. Let $M$ be an $R$-module and $N_{1}, N_{2} \in \operatorname{Min}(M)$ be respectively $\mathfrak{p}$-prime and $\mathfrak{q}$-prime submodules. Then $N_{1} \neq N_{2}$ if and only if $\mathfrak{p} \neq \mathfrak{q}$.

Proof. If $\mathfrak{p} \neq \mathfrak{q}$ then obviously $N_{1} \neq N_{2}$. Conversely, Let $N_{1} \neq N_{2}$ but $\mathfrak{p}=\mathfrak{q}$. Since

$N_{1}=\bigcap_{L \in \operatorname{Spec}_{R}^{\mathfrak{p}}(M)} L$ and $N_{2}=\bigcap_{L \in \operatorname{Spec}_{R}^{\mathfrak{q}}(M)} L$, it follows that $N_{1}=N_{2}$ which is a contradiction. $\square$

Definition 3.2. Let $M$ be an $R$-module and $B$ be a submodule of $M$. Set

$$
D(B):=\{N \in \operatorname{Min}(B): N \text { is not } a \text { finitely generated } R-\text { module }\} .
$$

Definition 3.3. Let $R$ be a Noetherian ring and $M$ be a finitely generated $R$-module. Then we define $\operatorname{dim}_{\operatorname{Spec}}(M)$ as:

$$
\operatorname{dimSpec}_{R}(M):=\sup \left\{\mathrm{ht}(N): N \in \operatorname{Spec}_{R}(M)\right\} .
$$

The minimal prime submodules of an $R$-module $M$ has been studied in [17], for example see [17, Theorem 2.1]. In the next theorem we present a new conditions that an $R$-module $M$ has only a finite number of minimal prime submodules, whenever $R$ is a Noetherian ring, which is a generalization of [3, Theorem 2.1]. 
Alg. Struc. Appl. Vol. 5 No. 1 (2018) 41-49.

Theorem 3.4. Let $R$ be a Noetherian ring, $M$ be an $R$-module and $B$ be a submodule of $M$. Then the following statements are equivalent:

(1) $\operatorname{Min}(B)$ is finite.

(2) For every $\mathfrak{P} \in \operatorname{Min}(B)$ there exists a finitely generated submodule $K_{\mathfrak{P}}$ of $\mathfrak{P}$ such that $\left|V\left(K_{\mathfrak{P}}\right) \bigcap \operatorname{Min}(B)\right|<\infty$.

(3) For every $\mathfrak{P} \in \operatorname{Min}(B)$ there exists a finitely generated submodule $N_{\mathfrak{P}}$ of $\mathfrak{P}$ such that $V\left(N_{\mathfrak{P}}\right) \bigcap \operatorname{Min}(B)=\{\mathfrak{P}\}$.

(4) For every $\mathfrak{P} \in \operatorname{Min}(B), \mathfrak{P} \nsubseteq \bigcup_{L \in \operatorname{Min}(B) \backslash\{\mathfrak{P}\}} L$.

(5) For every $\mathfrak{P} \in \operatorname{Min}(B)$ there exists an element $x_{\mathfrak{P}} \in \mathfrak{P}$ such that $V\left(R x_{\mathfrak{P}}\right) \cap \operatorname{Min}(B)=\{\mathfrak{P}\}$.

(6) For every $\mathfrak{P} \in D(B), \mathfrak{P} \nsubseteq \bigcup_{L \in \operatorname{Min}(B) \backslash\{\mathfrak{P}\}} L$.

(7) For every $\mathfrak{P} \in D(B)$ there exists an element $x_{\mathfrak{P}} \in \mathfrak{P}$ such that $V\left(R x_{\mathfrak{P}}\right) \bigcap \operatorname{Min}(B)=\{\mathfrak{P}\}$.

(8) For every $\mathfrak{P} \in D(B)$ there exists a finitely generated submodule $K_{\mathfrak{P}}$ of $\mathfrak{P}$ such that $\left|V\left(K_{\mathfrak{P}}\right) \bigcap \operatorname{Min}(B)\right|<\infty$.

(9) For every $\mathfrak{P} \in D(B)$ there exists a finitely generated submodule $N_{\mathfrak{P}}$ of $\mathfrak{P}$ such that $V\left(N_{\mathfrak{P}}\right) \bigcap \operatorname{Min}(B)=\{\mathfrak{P}\}$.

Proof. Without loss of generality, we may assume that $B=0, \operatorname{Spec}_{R}(M) \neq \emptyset$ and consequently $\operatorname{Min}(M) \neq \emptyset . \quad(1) \Rightarrow(2)$ Since $\operatorname{Min}(M)$ is finite, by Lemma 3.1 and Proposition 2.1, for every $\mathfrak{P} \in \operatorname{Min}(M), \mathfrak{P} \nsubseteq \bigcup_{L \in \operatorname{Min}(M) \backslash\{\mathfrak{P}\}} L$ and so there exists $x \in \mathfrak{P} \backslash \bigcup_{L \in \operatorname{Min}(M) \backslash\{\mathfrak{P}\}} L$. Set $K_{\mathfrak{P}}=R x$. Then $K_{\mathfrak{P}}$ is finitely generated and the set $V\left(K_{\mathfrak{P}}\right) \bigcap \operatorname{Min}(M)=\{\mathfrak{P}\}$ is finite.

$(2) \Rightarrow(3)$ Let $\mathfrak{P} \in \operatorname{Min}(M)$ and $V\left(K_{\mathfrak{P}}\right) \bigcap \operatorname{Min}(M)=\left\{\mathfrak{P}, \mathfrak{P}_{2}, \ldots, \mathfrak{P}_{n}\right\}$. Using Lemma 2.1 and Proposition 2.1 we can find an element $x \in \mathfrak{P} \backslash \bigcup_{i=2}^{n} \mathfrak{P}_{i}$. Let $N_{\mathfrak{P}}:=K_{\mathfrak{P}}+R x$. Then $N_{\mathfrak{P}}$ is finitely generated and $V\left(N_{\mathfrak{P}}\right) \bigcap \operatorname{Min}(M)=\{\mathfrak{P}\}$.

$(3) \Rightarrow(1)$ Suppose the contrary be true. Then the set $\operatorname{Min}(M)$ is infinite. Let

$$
A:=\left\{\mathfrak{p} \in \operatorname{Spec}(R): \operatorname{Spec}_{R}^{\mathfrak{p}}(M) \cap \operatorname{Min}(M) \neq \emptyset\right\}
$$

$E:=\{N \leq M: N$ is finitely generated and $V(N) \cap \operatorname{Min}(M)$ is a finite set. $\}$

$$
F:=\{L \leq M: \forall N \in E, N \nsubseteq L\}
$$

We show that there exists a maximal element $K$ of $F$ such that $\left(K:_{R} M\right)$ is a prime ideal. Since $\operatorname{Min}(M)$ is infinite, so the zero submodule of $M$ belong to the $F$ and therefore by Zorn's Lemma $F$ has a maximal element. Let $L$ be a maximal element of $F$. If $\left(L:_{R} M\right)$ be a prime ideal, we are through. If not, then it is clear that $\left(L:_{R} M\right) \neq R$. Let $\mathfrak{q}_{1} \in \operatorname{Ass}_{R}\left(R /\left(L:_{R} M\right)\right)$. By the definition there exists $r \in R \backslash\left(L:_{R} M\right)$ such that $\mathfrak{q}_{1}=\left(\left(L:_{R} M\right): r\right)$ and therefore $\mathfrak{q}_{1} r M \subseteq L$. Since $r \notin\left(L:_{R} M\right)$, it follows that there exists an element $x \in M$ such that 
$r x \notin L$. Now there exists $N \in E$ such that $N \subseteq L+R r x$. In particular,

$$
\mathfrak{q}_{1} N \subseteq L+\mathfrak{q}_{1} r x \subseteq L+\mathfrak{q}_{1} r M \subseteq L
$$

Since $\mathfrak{q}_{1} N$ is finitely generated, so $\left|V\left(\mathfrak{q}_{1} N\right) \cap \operatorname{Min}(M)\right|=\infty$. But in this case for all $\mathfrak{P} \in$ $\left(V\left(\mathfrak{q}_{1} N\right) \cap \operatorname{Min}(M)\right) \backslash(V(N) \cap \operatorname{Min}(M))$, we have $\mathfrak{q}_{1} N \subseteq \mathfrak{P}$ and $N \nsubseteq \mathfrak{P}$. Now if $\mathfrak{P}$ be a $\mathfrak{p}$ Prime submodule, then $\mathfrak{q}_{1} \subseteq \mathfrak{p}$ and so $\left|V\left(\mathfrak{q}_{1}\right) \cap A\right|=\infty$. Hence $\left|V\left(\mathfrak{q}_{1} M\right) \cap \operatorname{Min}(M)\right|=\infty$. So for all $N \in E$, we have $N \nsubseteq \mathfrak{q}_{1} M$ and therefore $\mathfrak{q}_{1} M \in F$. Let

$$
U:=\left\{\mathfrak{q} \in V\left(\mathfrak{q}_{1}\right): \mathfrak{q} M \in F\right\}
$$

Since $R$ is Noetherian it follows that $U$ has a maximal element, say $\mathfrak{q}_{2}$. But $\mathfrak{q}_{2} M \subseteq H$, for some maximal element $H$ of $F$. We claim that $\left(H:_{R} M\right)$ is a prime ideal of $R$. If not, according to the above argument, there exists $\mathfrak{q}_{3} \in \operatorname{Ass}_{R}\left(R /\left(H:_{R} M\right)\right)$ such that $\mathfrak{q}_{3} M \in F$ and $\mathfrak{q}_{2} \subseteq(H: R M) \subseteq \mathfrak{q}_{3}$. By choosing $\mathfrak{q}_{2}$, we must have $\mathfrak{q}_{2}=\mathfrak{q}_{3}$, which is a contradiction. Therefore $\left(H:_{R} M\right)=\mathfrak{q}_{2}$ is a prime ideal. Now we show that $H$ is a $\mathfrak{q}_{2}$-prime submodule. Otherwise there exist $x \in M \backslash H$ and $r \in R \backslash \mathfrak{q}_{2}$, such that $r x \in H$. So $r \in Z_{R}(M / H)=\bigcup_{\mathfrak{q} \in \operatorname{Ass}_{R}(M / H)} \mathfrak{q}$ and hence there exists $\mathfrak{q}^{\prime} \in \operatorname{Ass}_{R}(M / H)$ such that $r \in \mathfrak{q}^{\prime}$. Consequently, $\mathfrak{q}_{2} \subset \mathfrak{q}^{\prime}$. On the other hand by the definition $\mathfrak{q}^{\prime}=\left(H:_{R} y\right)$ for some $y \in M \backslash H$. Since $H \subset H+R y$, it follows that there exists $N \in E$ such that $N \subseteq H+R y$ and so $\mathfrak{q}^{\prime} N \subseteq H$. According to the above argument, $\left|V\left(\mathfrak{q}^{\prime} M\right) \cap \operatorname{Min}(M)\right|=\infty$ which implies $\mathfrak{q}^{\prime} M \in F$. Finally, we have $\mathfrak{q}_{2}=\left(H:_{R} M\right) \subset \mathfrak{q}^{\prime}$, which is a contradiction with the choosing $\mathfrak{q}_{2}$. Therefore $H$ is a $\mathfrak{q}_{2}$-prime submodule of $M$. Whence, $H$ contains a minimal prime submodule of $M$ such as $\mathfrak{P}$. By assumption there exists a submodule $N_{\mathfrak{P}}$ of $\mathfrak{P}$ such that $N_{\mathfrak{P}} \subseteq \mathfrak{P} \subseteq H$ and $N_{\mathfrak{P}} \in E$, which is a contradiction. Therefore, $\operatorname{Min}(M)$ is a finite set.

Now the proof of $(1) \Leftrightarrow(2) \Leftrightarrow(3)$ is complete.

$(1) \Rightarrow(4)$ Follows from Lemma 3.1 and Proposition 2.1.

$(4) \Rightarrow(1) \Leftrightarrow(5)$ Since $(5) \Leftrightarrow(4) \Rightarrow(3)$ is clear so we have $(1) \Leftrightarrow(4) \Leftrightarrow(5)$.

Now we have the following: $(1) \Leftrightarrow(2) \Leftrightarrow(3) \Leftrightarrow(4) \Leftrightarrow(5)$.

$(4) \Rightarrow(6)$ Is clear.

$(6) \Rightarrow(3)$ Since for every $\mathfrak{P} \in D(0), \mathfrak{P} \nsubseteq \bigcup_{L \in \operatorname{Min}(M) \backslash\{\mathfrak{P}\}} L$, it follows that there exists $x_{\mathfrak{P}} \in \mathfrak{P}$ such that $V\left(R x_{\mathfrak{P}}\right) \cap \operatorname{Min}(M)=\{\mathfrak{P}\}$. On the other hand for all $\mathfrak{P} \in(\operatorname{Min}(M) \backslash D(0))$, we have $V(\mathfrak{P}) \cap \operatorname{Min}(M)=\{\mathfrak{P}\}$, where $\mathfrak{P}$ is finitely generated. So the assertion follows.

$(6) \Leftrightarrow(7)$ and $(1) \Rightarrow(8),(9)$ are clear.

$(8),(9) \Rightarrow(3)$ Follow by a similar arguments as in $(6) \Rightarrow(3)$.

The following results follow from Theorem 3.4. 
Alg. Struc. Appl. Vol. 5 No. 1 (2018) 41-49.

Corollary 3.5. Let $R$ be a Noetherian ring, $M$ an $R$-module and $B$ be a proper submodule of $M$. Then $\operatorname{Min}(B)$ is infinite if and only if there exists $\mathfrak{P} \in D(B)$ such that $\mathfrak{P} \subseteq \bigcup_{L \in(\operatorname{Min}(B) \backslash\{\mathfrak{P}\})} L$.

Proof. Follows immediately from Theorem 3.4. $\square$

Corollary 3.6. Let $R$ be a Noetherian ring, $M$ an $R$-module and $B$ be a proper submodule of $M$ such that any minimal prime submodule over $B$ is finitely generated. Then $\operatorname{Min}(B)$ is finite.

Proof. Follows immediately from Theorem 3.4. $\square$

Definition 3.7. Let $R$ be a Noetherian ring, $M \neq 0$ be a finitely generated $R$-module and $N$ be a proper submodule of $M$. Then the radical of $N$ is defined as:

$$
\operatorname{Rad}(N)=\bigcap_{L \in \operatorname{Min} N} L
$$

Before bringing the next definition, recall that for any ideal $I$ of a Noetherian ring, the arithmetic rank of $I$, denoted by $\operatorname{ara}(I)$, is the least number of elements of $I$ required to generate an ideal which has the same radical as $I$, i.e.,

$$
\operatorname{ara}(I):=\min \left\{n \in \mathbb{N}_{0}: \exists x_{1}, \ldots, x_{n} \in I \text { with } \operatorname{Rad}\left(\left(x_{1}, \ldots, x_{n}\right)\right)=\operatorname{Rad}(I)\right\}
$$

Definition 3.8. Let $R$ be a Noetherian ring, $M \neq 0$ be a finitely generated $R$-module and $N$ be a proper submodule of $M$. We define the arithmetic rank of $N$, as:

$$
\operatorname{ara}(N):=\min \left\{n \in \mathbb{N}_{0}: \exists x_{1}, \ldots, x_{n} \in N \text { with } \operatorname{Rad}\left(\left(x_{1}, \ldots, x_{n}\right)\right)=\operatorname{Rad}(N)\right\}
$$

The next theorem is a generalization of [15, Theorem 2.7].

Theorem 3.9. Let $R$ be a Noetherian ring, $M \neq 0$ a finitely generated $R$-module and $N$ be a proper submodule of $M$. Then $\operatorname{ara}(N) \leq \operatorname{dimSpec}_{R}(M)+1$

Proof. Let $d:=\operatorname{dimSpec}_{R}(M)$. We may assume that $d$ is finite. Now, suppose, to the contrary, that $\operatorname{ara}(N)>d+1$. Let $n:=\operatorname{ara}(N)$. Since $n>d+1 \geq 1$ it follows from the definition that there exist elements $x_{1}, \ldots, x_{n}$ in $N$ such that $\operatorname{Rad}(N)=\operatorname{Rad}\left(\left(x_{1}, \ldots, x_{n}\right)\right)$. As $n>0$ it follows that $\operatorname{Min}(0) \backslash V(N) \neq \emptyset$. Therefore it follows from Lemma 3.1 and proposition 2.1 that 
$N \nsubseteq \bigcup_{L \in \operatorname{Min}(0) \backslash V(N)} L$. Therefore $\left(x_{1}, \ldots, x_{n}\right) \nsubseteq \bigcup_{L \in \operatorname{Min}(0) \backslash V(N)} L$, and so by Proposition 2.2 there is $a_{1} \in\left(x_{2}, \ldots, x_{n}\right)$ such that

$$
x_{1}+a_{1} \notin \bigcup_{L \in \operatorname{Min}(0) \backslash V(N)} L
$$

Let $y_{1}:=x_{1}+a_{1}$. Then $y_{1} \in N$ and $\operatorname{Rad}(N)=\operatorname{Rad}\left(\left(y_{1}, x_{2}, \ldots, x_{n}\right)\right)$. We shall construct the sequence $y_{1}, \ldots, y_{n-1} \in N$ such that $\operatorname{Rad}(N)=\operatorname{Rad}\left(\left(y_{1}, \ldots, y_{n-1}, x_{n}\right)\right)$ and $y_{j} \notin \bigcup_{L \in \operatorname{Min}\left(\left(y_{1}, \ldots, y_{j-1}\right) \backslash V(N)\right.} L$, for each $1 \leq j \leq n-1$, by an inductive process. To do this end, assume that $1 \leq k<n-1$, and that we have already constructed elements $y_{1}, \ldots, y_{k}$ such that

$$
\operatorname{Rad}(N)=\operatorname{Rad}\left(\left(y_{1}, \ldots, y_{k}, x_{k+1}, \ldots, x_{n}\right)\right)
$$

We show how to construct $y_{k+1}$. To do this, as $k<n-1$ it follows that

$$
\operatorname{Min}\left(\left(y_{1}, \ldots, y_{k}\right)\right) \backslash V(N) \neq \emptyset \text {. }
$$

Therefore it follows from Lemma 3.1 and proposition 2.1 that

$$
N \nsubseteq \underset{L \in \operatorname{Min}\left(\left(y_{1}, \ldots, y_{k}\right)\right) \backslash V(N)}{\bigcup} L
$$

Therefore $\left(y_{1}, \ldots, y_{k}, x_{k+1}, \ldots, x_{n}\right) \nsubseteq \subseteq \bigcup_{L \in \operatorname{Min}\left(\left(y_{1}, \ldots, y_{k}\right)\right) \backslash V(N)} L$, and so by Proposition 2.2 there is $a_{k+1} \in\left(y_{1}, \ldots, y_{k}, x_{k+2}, \ldots, x_{n}\right)$ such that

$$
x_{k+1}+a_{k+1} \notin \underset{L \in \operatorname{Min}\left(\left(y_{1}, \ldots, y_{k}\right)\right) \backslash V(N)}{\bigcup} L .
$$

Let $y_{k+1}:=x_{k+1}+a_{k+1} . \quad$ Then $y_{k+1} \in N$ and $\operatorname{Rad}(N)=\operatorname{Rad}\left(\left(y_{1}, \ldots, y_{k}, y_{k+1}, x_{k+2} \ldots, x_{n}\right)\right)$. This completes the inductive step in the construction. Now it is easy to see that $\operatorname{Min}\left(\left(y_{1}, \ldots, y_{n-1}\right)\right) \backslash V(N) \neq \emptyset$. Also, by using an induction argument we can deduce that for any $1 \leq j \leq n-1$ and any $L \in \operatorname{Min}\left(\left(y_{1}, \ldots, y_{j}\right)\right) \backslash V(N)$ we have $\operatorname{ht}(L) \geq j$. Consequently, since there exists a prime submodule $L$ of $M$ in which $L \in \operatorname{Min}\left(\left(y_{1}, \ldots, y_{n-1}\right)\right) \backslash V(N)$ it follows that $n-1 \leq \operatorname{ht}(L) \leq \operatorname{dimSpec}_{R}(M)=d$, which implies that $n \leq d+1$, as required. $\square$

\section{Acknowledgments}

The author is deeply grateful to the referee for a very careful reading of the manuscript and many valuable suggestions. 


\section{REFERENCES}

[1] S. Abu-Saymeh, On dimensions of finitely generated modules, Comm. Alg. 23(1995), 1131-1144.

[2] J. Azami and M. Khajepour, Topics in prime submodules and other aspects of the prime avoidence theorem, Preprint(Mathematical Reports) .

[3] K. Bahmanpour, A. Khojali and R. Naghipour, A note on minimal prime divisors of an ideal, Algebra Colloq. 18(2011), 727-732.

[4] M. Behboodi, A generalization of the classical Krull dimension for modules, J. Algebra. 305(2006), 11281148.

[5] W. Bruns and J. Herzog, Cohen-Macaulay Rings, Cambridge Univ. Press, Cambridge, UK, 1993.

[6] J. Dauns, Prime modules, J. Reine Anegew. Math. 298(1978), 156-181.

[7] J. Jenkins and P. F. Smith, On the prime radical of a module over commutative ring, Comm. Alg. 20(1992), 3593-3602.

[8] O. A. S. Karamzadeh, The Prime Avoidance Lemma revisited, Kyungpook Math. J. 52(2012), 149-153.

[9] C. P. Lu, Unions of prime submodules, Houston J. Math. 23, no.2(1997), 203-213.

[10] K. H. Leung and S. H. Man, On commutative Noetherian rings which satisfy the radical formula, Glasgow math. J. 39(1997), 285-293.

[11] S. H. Man and P. F. Smith, On chains of prime submodules, Israel J. Math. 127(2002), 131-155.

[12] A. Marcelo and J. Munoz Maque, Prime submodules, the discent invariant, and modules of finite length, J. Algebra 189(1997), 273-293.

[13] H. Matsumura, Commutative ring theory, Cambridge Univ. Press, Cambridge, UK, 1986.

[14] R. L. McCasland and P. F. Smith, Prime submodules of Noetherian modules, Rocky Mountain J. Math. 23(1993), 1041-1062.

[15] A. A. Mehrvarz, K. Bahmanpour and R. Naghipour, Arithmetic rank, cohomological dimension and filter regular sequences, J. Alg. Appl. 8(2009), 855-862.

[16] J. J. Rotman, An introduction to homological algebra, Pure Appl. Math., Academic Press, New York, 1979.

[17] D. Pusat-Yilmaz and P. F. Smith, Chain conditions in modules with krull dimension, Comm. Alg. 24(13)(1996), 4123-4133.

\section{Jafar A'zami}

Department of mathematics, Faculty of sciences

University of Mohaghegh Ardabili, Ardabil

Ardabil, Iran.

jafar.azami@gmail.com 